\title{
Caracterização Estrutural de Nanocompósitos de Blendas HDPE/LLDPE e OMMT Obtidos por Diferentes Sequências de Mistura
}

\author{
Fabio R. Passador, Adhemar C. Ruvolo Filho, Luiz A. Pessan \\ Departamento de Engenharia de Materiais, UFSCar
}

\begin{abstract}
Resumo: Nanocompósitos de blendas de polietileno de alta densidade (HDPE) com polietileno linear de baixa densidade (LLDPE) e OMMT (montmorilonita organofílica) foram preparados em um reômetro de torque, utilizando-se como sistema compatibilizante uma mistura de HDPE-g-MA e LLDPE-g-MA, ambos com 1\% de anidrido maleico. O efeito de cinco sequências de mistura na formação da microestrutura dos nanocompósitos foi estudado. A caracterização estrutural foi realizada por análises de difração de raio X de alto ângulo (WAXD), microscopia eletrônica de transmissão (MET) e comportamento reológico em regime permanente de deformação. Os resultados mostraram que a formação da microestrutura depende da ordem de mistura dos componentes e a utilização de mistura de dois agentes compatibilizantes miscíveis com os constituintes da matriz auxiliou na distribuição da polaridade, facilitando a dispersão das nanoargilas por toda a matriz. Dentre as sequências estudadas, as que a nanoargila foi primeiramente misturada com componentes de menor viscosidade e cristalinidade (LLDPE e/ou LLDPE-g-MA) apresentaram melhor dispersão e distribuição da nanoargila na blenda polimérica.
\end{abstract}

Palavras-chave: Nanocompósitos, HDPE/LLDPE, sequência de mistura.

\section{Structural Characterization of HDPE/LLDPE and OMMT Blend-Based Nanocomposites Obtained by Different Blending Sequences}

Abstract: High density polyethylene/linear low density polyethylene (HDPE/LLDPE) blend-based nanocomposites were prepared in a torque rheometer, using a mixture of HDPE-g-MA and LLDPE-g-MA both containing $1 \%$ of maleic anhydride as compatibilizer system. The effect from five blending sequences on the microstructure of the nanocomposites was investigated. The structural characterization was performed through wide angle X-ray diffraction (WAXD), transmission electron microscopy (TEM) and rheological properties. The results showed that the formation of morphology is dependent on the sequence of nanocomposite preparation and that the use of a mixture of two compatibilizer agents, miscible with both components of the blend matrix, facilitated the dispersion of the nanoclay throughout the matrix. The blending sequence where the nanoclay was first mixed with components of lower viscosity and crystallinity (LLDPE and/or LLDPE-g-MA) yielded better distribution and dispersion of nanoclay in the polymer blend.

Keywords: Nanocomposites, HDPE/LLDPE, blend sequence.

\section{Introdução}

Poliolefinas, como polietilenos e polipropileno, são amplamente utilizadas em aplicações para embalagens, no setor automotivo e elétrico, onde as propriedades de resistência térmica, mecânica e de permeação a gases possuem grande importância. As propriedades finais dos produtos obtidos a partir de poliolefinas estão intimamente relacionadas à morfologia desses materiais desenvolvida durante o processamento. A grande versatilidade de uso e baixo custo das poliolefinas têm gerado grande interesse tecnológico e científico, principalmente na obtenção de blendas HDPE/LLDPE ${ }^{[1-6]}$. O processamento de HDPE com baixo índice de fluidez é dificultado devido a elevada viscosidade do fundido. A adição de um material com estrutura química semelhante como o LLDPE, com alto índice de fluidez, auxilia no processamento, reduzindo a viscosidade do fundido.

Dentre as alternativas utilizadas para melhorar o desempenho mecânico e de barreira das poliolefinas, a dispersão de camadas de silicatos lamelares tem se mostrado bastante atraente. Estudos recentes ${ }^{[7-10]}$ têm mostrado que a modificação orgânica da superfície das argilas, que são hidrofílicas, com íons alquilamônio pode auxiliar no processo de esfoliação das lamelas de argila em polímeros polares, como as poliamidas, utilizando condições e técnicas de processamento apropriados. Entretanto, é difícil a obtenção de nanocompósitos esfoliados de poliolefinas devido ao caráter hidrofóbico destas resinas e ao baixo nível de interações com a superfície polar dos silicatos. Dessa forma, apenas a aplicação de um alto cisalhamento durante o processo de fusão dificilmente levará a delaminação dos tactóides de argila na matriz de polietileno. Basicamente, dois tipos de procedimentos têm sido descritos na literatura para contornar essa dificuldade: o primeiro envolve o método de polimerização in situ ${ }^{[11,12]}$, com a possibilidade de ocorrer re-ordenação das camadas da argila e perda da estrutura esfoliada quando a resina é processada; o segundo procedimento envolve a mistura no estado fundido, sendo necessário melhorar a interação da carga inorgânica com a matriz polimérica através da utilização de agentes compatibilizantes dentre as quais se destaca a utilização de resinas poliméricas modificadas (enxertadas) com grupos polares, principalmente grupos anidrido maleico, que proporcionam um aumento na afinidade química e estrutural da argila com a matriz polimérica ${ }^{[13-17]}$.

Além do tipo de argila organofílica e compatibilizantes utilizados para a obtenção de estrutura intercalada/esfoliada em nanocompósitos de polietileno/argila, a sequência de mistura dos componentes constituintes do sistema também pode ter um efeito significativo na formação da microestrutura ${ }^{[18-20]}$, possibilitando localizar preferencialmente a nanoargila em uma das fases do sistema e, desta forma, influenciar diretamente nas propriedades dos nanocompósitos. O objetivo principal deste trabalho foi estudar o efeito da incorporação de dois agentes compatibilizantes

Autor para correspondência: Fabio R. Passador, Departamento de Engenharia de Materiais, Universidade Federal de São Carlos - UFSCar,

Rod. Washington Luis, Km 235, CP 676, CEP 13565-905, São Carlos, SP, Brasil, e-mail: fabiopassador@gmail.com 
enxertados com anidrido maleico, escolhidos pela semelhança estrutural e por possuírem índices de fluidez semelhantes aos materiais utilizados como matriz polimérica, e também estudar os efeitos da ordem de mistura dos componentes de nanocompósitos da blenda HDPE/LLDPE na formação da morfologia destes sistemas.

\section{Experimental}

\section{Materiais}

O polietileno de alta densidade (HDPE) com índice de fluidez de $0,38 \mathrm{~g} / 10 \mathrm{~min}\left(190{ }^{\circ} \mathrm{C} / 2,16 \mathrm{~kg}\right)$, de especificação DMDA $6200 \mathrm{NT}-7$ foi fornecida pela Dow Chemical. O polietileno linear de baixa densidade (LLDPE) é um copolímero de buteno-1, produzido pelo processo de solução com índice de fluidez de $29 \mathrm{~g} / 10 \mathrm{~min}$ $\left(190{ }^{\circ} \mathrm{C} / 2,16 \mathrm{~kg}\right.$ ), de especificação IC-32 foi fornecida pela Braskem. O número de ramificações para os polímeros puros foi determinado por Ressonância Magnética Nuclear de Carbono ${ }^{13} \mathrm{C}$ baseada nos trabalhos de Fan $^{[21]}$ e Spevacek ${ }^{[22]}$. O HDPE possui aproximadamente 3 ramificações por 1000 átomos de carbono, enquanto que o LLDPE possui 13 ramificações por 1000 átomos de carbono.

O sistema de compatibilização consistiu da mistura de polietileno de alta densidade enxertado com anidrido maleico (HDPE-g-MA) com MFI = 3-6 g/10 min $\left(190{ }^{\circ} \mathrm{C} / 2,16 \mathrm{~kg}\right)$, de especificação Polybond $^{\circledR} 3009$ e polietileno linear de baixa densidade enxertado com anidrido maleico (LLDPE-g-MA) com MFI = $29 \mathrm{~g} / 10 \mathrm{~min}$ $\left(190{ }^{\circ} \mathrm{C} / 2,16 \mathrm{~kg}\right)$, de especificação Polybond ${ }^{\circledR} 3109$, ambos da Crompton Corporation e funcionalizados com $1 \%$ em massa de anidrido maleico. Esses compatibilizantes foram escolhidos por possuírem índices de fluidez semelhantes aos materiais utilizados como matriz polimérica e, assim, espera-se que os agentes compatibilizantes e os polímeros utilizados sejam miscíveis uns com os outros e a blenda HDPE/LLDPE compatibilizada tenha melhor afinidade com o modificador orgânico da nanoargila. A nanocarga utilizada foi a montmorilonita natural modificada com um sal quaternário de amônio (OMMT), de nome comercial Cloisite ${ }^{\circledR}$ 20A produzida pela Southern Clay Products Inc., com densidade aparente de aproximadamente $0,32 \mathrm{~g} . \mathrm{cm}^{-3}$, com perda de massa durante a queima de $38 \%$ e com espaçamento basal de $2,32 \mathrm{~nm}$. O tratamento da argila foi realizado com surfactante apolar e possui alto grau de hidrofobicidade. O teor de nanocarga adicionada nos sistemas foi corrigido considerando a perda de massa.

\section{Preparação dos nanocompósitos}

Os nanocompósitos foram preparados através de mistura no estado fundido, em rêometro de torque Haake, modelo Rheomix 600 p, com rotores tipo roller, operando-se a uma temperatura de $180{ }^{\circ} \mathrm{C}$ e $80 \mathrm{rpm}$ por 10 minutos. Para estudar o efeito da adição da mistura de HDPE-g-MA e LLDPE-g-MA na compatibilização de nanocompósitos de blenda HDPE/LLDPE e a influência desse sistema nas propriedades reológicas, no grau de cristalinidade e na morfologia desses nanocompósitos, cinco sequências de mistura foram preparadas:

- M1. (HDPE + LLDPE + HDPE-g-MA + LLDPE-g$M A+O M M T)$ - mistura simultânea dos componentes;

- $M 2$. (LLDPE + LLDPE-g-MA + OMMT) + HDPE + HDPE-g$M A$ - preparação do nanocompósito de LLDPE compatibilizado com LLDPE-g-MA e posterior mistura com HDPE e HDPE-gMA;

- M3. $(H D P E+H D P E-g-M A+O M M T)+L L D P E+L L D P E-g-$ $M A$ - preparação do nanocompósito de HDPE compatibilizado com HDPE-g-MA e posterior mistura com LLDPE e LLDPE-g-MA;
- M4. (HDPE + HDPE-g-MA + LLDPE + LLDPE-g$M A)+O M M T$ - preparação da blenda compatibilizada e posterior adição da nanoargila;

- M5. (HDPE-g-MA + LLDPE-g-MA + OMMT) + HDPE + $L L D P E$ - preparação do concentrado HDPE-g-MA/LLDPE-gMA/nanoargila e posterior diluição no HDPE e LLDPE.

Após cada etapa de pré-mistura, exceto para a primeira onde os componentes foram adicionados simultaneamente em uma única etapa, o material obtido foi moído para novamente ser submetido à mistura no estado fundido.

Os nanocompósitos obtidos através das diferentes sequências de mistura possuem razão HDPE/LLDPE de 3:1, com 5\% em massa do sistema compatibilizante e $2,5 \%$ em massa de nanocarga. Os materiais puros e a blenda HDPE/LLDPE (75/25\%) também foram preparados nas mesmas condições.

\section{Avaliação da miscibilidade da blenda HDPE/LLDPE}

A miscibilidade da blenda HDPE/LLDPE (75/25\%) foi avaliada através de análise dinâmico-mecânica (DMA) e análises de calorimetria exploratória diferencial (DSC).

A análise dinâmico-mecânica foi realizada em um equipamento de DMA da TA Instruments, modelo Q800. Os corpos de prova utilizados foram prensados à $180^{\circ} \mathrm{C}$ por 3 minutos após mistura em reômetro de torque, com espessura de $1 \mathrm{~mm}$. O equipamento DMA foi operado no modo flexão em três pontos, a uma frequência de $1 \mathrm{~Hz}$, taxa de aquecimento de $2{ }^{\circ} \mathrm{C} / \mathrm{min}$ e intervalo de temperatura de -130 a $110^{\circ} \mathrm{C}$.

Para as análises de DSC, utilizou-se o equipamento da TA Instruments, modelo QS100 com nitrogênio como gás de arraste, em fluxo contínuo de $50 \mathrm{~mL} / \mathrm{min}$. As amostras foram inicialmente aquecidas até $180{ }^{\circ} \mathrm{C}$ a uma taxa de aquecimento de $10{ }^{\circ} \mathrm{C} / \mathrm{min}$, permanecendo nesta temperatura por 5 minutos. A seguir foram resfriadas até $40{ }^{\circ} \mathrm{C}$ a uma taxa de $10{ }^{\circ} \mathrm{C} / \mathrm{min}$ para determinação da temperatura de cristalização $\left(\mathrm{T}_{c}\right)$ e novamente foram aquecidas até $180{ }^{\circ} \mathrm{C}$ a uma taxa de $10{ }^{\circ} \mathrm{C} / \mathrm{min}$. O valor da temperatura de fusão cristalina $\left(\mathrm{T}_{\mathrm{m}}\right)$ foi obtido no segundo ciclo de aquecimento.

\section{Caracterização estrutural}

O grau de intercalação/esfoliação da nanoargila e dos nanocompósitos foi avaliado através de análises de difração de raio $\mathrm{X}$ de alto ângulo (WAXD) e microscopia eletrônica de transmissão (MET).

Análises de WAXD foram realizadas utilizando-se um difratômetro Regaku GE/Gerflex Analix, com radiação $\mathrm{CuK} \alpha$ $(\lambda=1,54056 \AA)$, operado a $40 \mathrm{kV}$ e $25 \mathrm{~mA}$, com $2 \theta$ variando entre 1,6 e $30^{\circ}$, a uma taxa de $1 \% \mathrm{~min}$, em amostras prensadas com espessura de $0,2 \mathrm{~mm}$. Além das informações sobre o tipo de estrutura formado nos nanocompósitos, foram feitas análises para determinação da cristalinidade e do tamanho de cristalito das amostras estudadas.

A cristalinidade foi obtida através da deconvolução matemática dos difratogramas na região de $2 \theta$ variando entre 15 e $30^{\circ}$ e calculada através da Equação 1.

$$
X_{c}=\frac{\sum A_{\text {crist }}}{\sum A_{\text {crist }}+\sum A_{\text {amorfa }}}
$$

onde $\mathrm{A}_{\text {crist }}$ e $\mathrm{A}_{\text {amorfa }}$ são as áreas dos picos cristalinos e da banda amorfa, respectivamente. O tamanho aparente de cristalito foi determinado através da equação de Scherrer (Equação 2).

$$
D_{(h k l)}=\frac{K \lambda}{\beta \cos \theta}
$$


onde $\beta$ é a largura meia-altura do pico de difração (em radianos), $K=0,9, \theta$ é o ângulo de Bragg e $\lambda$ é o comprimento de onda do raio X. Foram calculados os valores de $D_{(h k l)}$ para o plano (110).

A morfologia dos nanocompósitos foi analisada pela observação de imagens obtidas em um microscópio eletrônico de transmissão. As amostras foram submetidas ao "trimming" (corte na forma de trapézio) e foram criogenicamente microtomadas, ou seja, cortadas em seções ultrafinas, $40 \mathrm{~nm}$ de espessura, com facas de diamante, por um micrótomo tipo Riechert-Jung Ultracut E, com temperatura da amostra entre $-65 \mathrm{e}-75^{\circ} \mathrm{C}$ e temperatura da faca em $-50{ }^{\circ} \mathrm{C}$. Foram utilizadas telas ou "grids" de cobre para coletar as amostras fatiadas que se encontravam imersas em uma solução de DMSO: água (3:2). Posteriormente, as amostras foram analisadas em microscópio eletrônico de transmissão Philips, modelo CM120, a uma voltagem de aceleração de $120 \mathrm{kV}$.

A caracterização reológica foi utilizada para verificar a presença de interações entre os componentes do sistema, através da comparação do comportamento dos materiais puros com a blenda e os nanocompósitos estudados.

O comportamento reológico foi avaliado através de ensaios de viscosidade em função da taxa de cisalhamento (regime permanente), em ambiente inerte de nitrogênio, na temperatura de $180{ }^{\circ} \mathrm{C}$, utilizando-se um reômetro de tensão controlada $\mathrm{AR}$ G2, da TA Instruments. A geometria de ensaio utilizada foi a de placas paralelas, com diâmetro de placa de $25 \mathrm{~mm}$ e distância entre as placas de $1 \mathrm{~mm}$. A deformação aplicada em cada ensaio foi definida de acordo com o material, assegurando que os ensaios fossem conduzidos em regiões de comportamento viscoelástico linear.

\section{Resultados e Discussão}

\section{Avaliação da miscibilidade da blenda HDPE/LLDPE através de DMA e DSC}

Uma das características importantes a ser considerada em uma blenda polimérica é a miscibilidade ou o grau de interação entre seus componentes, que corresponde ao nível de mistura molecular entre os polímeros ou fases constituintes do sistema. A miscibilidade ou o grau de interação entre as possíveis fases presentes no sistema determinam direta ou indiretamente o comportamento do novo material desenvolvido ${ }^{[23]}$. Um dos critérios mais aceitos e utilizados

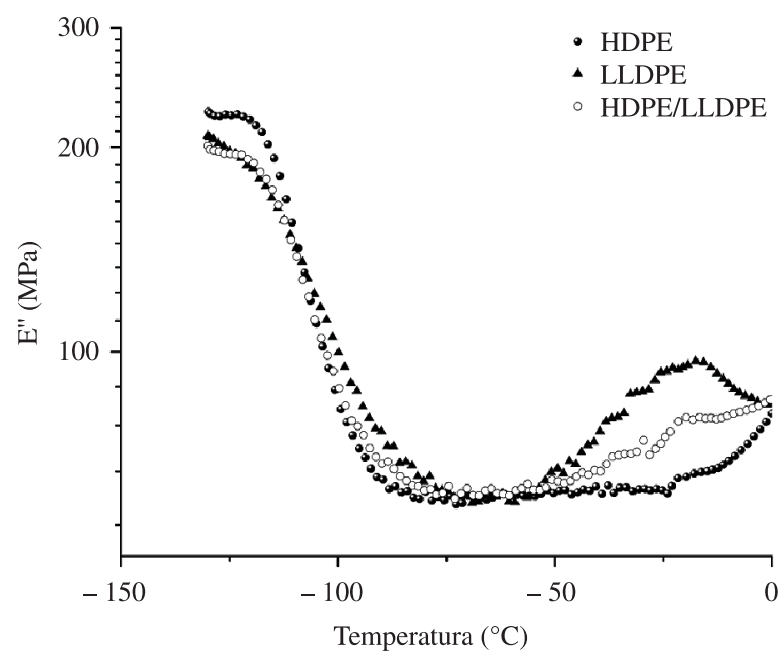

(a) para a avaliação da miscibilidade em blendas poliméricas é a detecção de uma única transição vítrea cuja temperatura se situa entre as transições vítreas dos componentes poliméricos individuais que constituem a blenda. A temperatura de transição vítrea $\left(\mathrm{T}_{\mathrm{g}}\right)$ é uma característica fundamental dos polímeros, pois representa um limite de temperatura, abaixo da qual a energia térmica não é suficiente para que as barreiras rotacionais em torno das ligações sejam superadas e, portanto, as mudanças conformacionais do polímero são inibidas ${ }^{[24]}$.

A Figura 1 apresenta os resultados da análise dinâmico-mecânica dos materiais puros e da blenda HDPE/LLDPE (75/25\%). Através desta análise foi possível observar a presença de uma única transição vítrea para a blenda HDPE/LLDPE, sendo a temperatura intermediária entre as temperaturas das transições vítreas dos materiais puros, apresentados na Tabela 1. Além disso, o valor da $\mathrm{T}_{\mathrm{g}}$ obtido experimentalmente $\left(-115,3{ }^{\circ} \mathrm{C}\right)$ está em concordância com o valor de $\mathrm{T}_{\mathrm{g}}$ calculado através da Equação de $\operatorname{Fox}^{[25]}\left(-115,8{ }^{\circ} \mathrm{C}\right)$, sugerindo que a blenda polimérica possivelmente seja miscível.

Porém, como as Tg dos materiais puros são muito próximas, foram realizados ensaios de DSC para verificação das temperaturas de fusão cristalina $\left(\mathrm{T}_{\mathrm{m}}\right)$ e cristalização $\left(\mathrm{T}_{\mathrm{c}}\right) \mathrm{e}$, assim, verificar o comportamento de cristalização da blenda, como um segundo critério para averiguação do estado de mistura da blenda polimérica. A Figura 2 apresenta os termogramas dos materiais puros e da blenda HDPE/LLDPE (75/25), obtidos por DSC, os valores da temperatura de fusão cristalina $\left(\mathrm{T}_{\mathrm{m}}\right)$ e temperatura de cristalização $\left(\mathrm{T}_{\mathrm{c}}\right)$ são apresentados na Tabela 1 .

A blenda polimérica apresentou uma única $\mathrm{T}_{\mathrm{m}}$ e $\mathrm{T}_{\mathrm{c}}$, indicando possível miscibilidade total dos elementos constituintes no estado fundido e, de acordo com a literatura ${ }^{[1-4]}$, sugere que esteja ocorrendo co-cristalização do HDPE com o LLDPE. Na co-cristalização, os dois polímeros formam cristais isomórficos, ou seja, as cadeias de

Tabela 1. Valores de temperatura de transição vítrea $(\mathrm{Tg})$, temperatura de cristalização (Tc) e temperatura de fusão cristalina (Tm) do HDPE, LLDPE e blenda HDPE/LLDPE (75/25\%).

\begin{tabular}{cccc}
\hline Amostra & $\mathbf{T}_{\mathrm{g}}\left({ }^{\circ} \mathbf{C}\right)$ & $\mathbf{T}_{\mathrm{m}}\left({ }^{\circ} \mathbf{C}\right)$ & $\mathbf{T}_{\mathbf{c}}\left({ }^{\circ} \mathbf{C}\right)$ \\
\hline HDPE & -116 & 134 & 115 \\
HDPE/LLDPE $(75 / 25 \%)$ & -115 & 131 & 115 \\
LLDPE & -114 & 124 & 106 \\
\hline
\end{tabular}

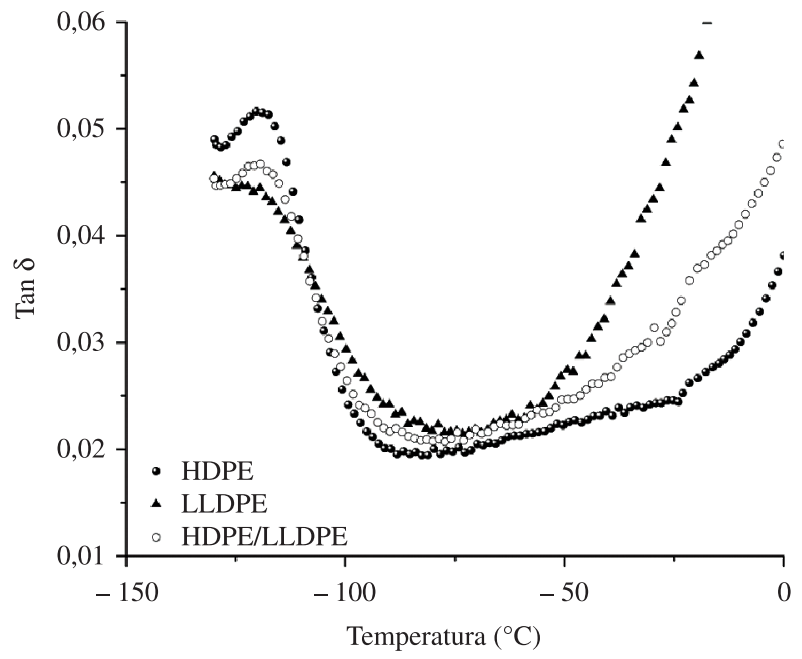

(b)

Figura 1. Curvas obtidas por análise dinâmico-mecânica dos materiais puros e da blenda HDPE/LLDPE (75/25\%): a) módulo de perda e b) tan $\delta$. 


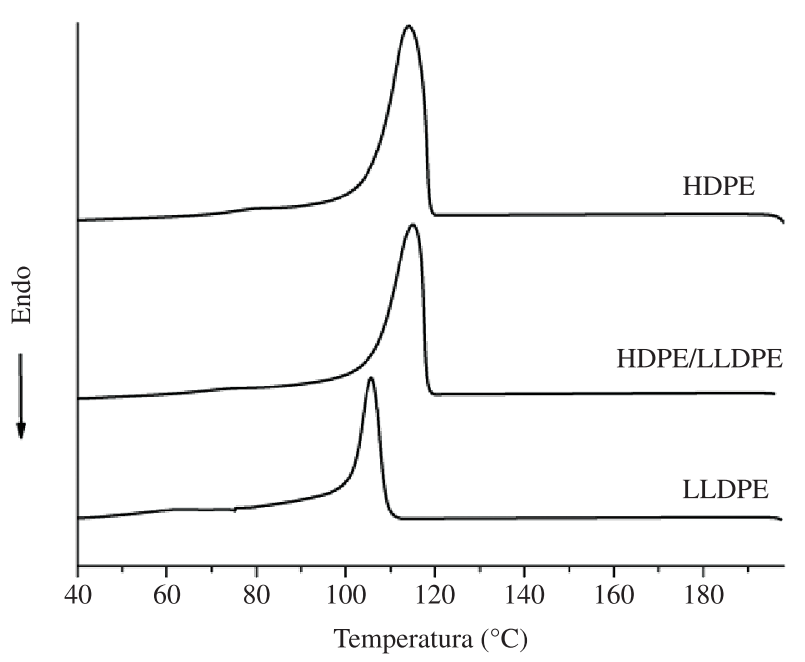

(a)

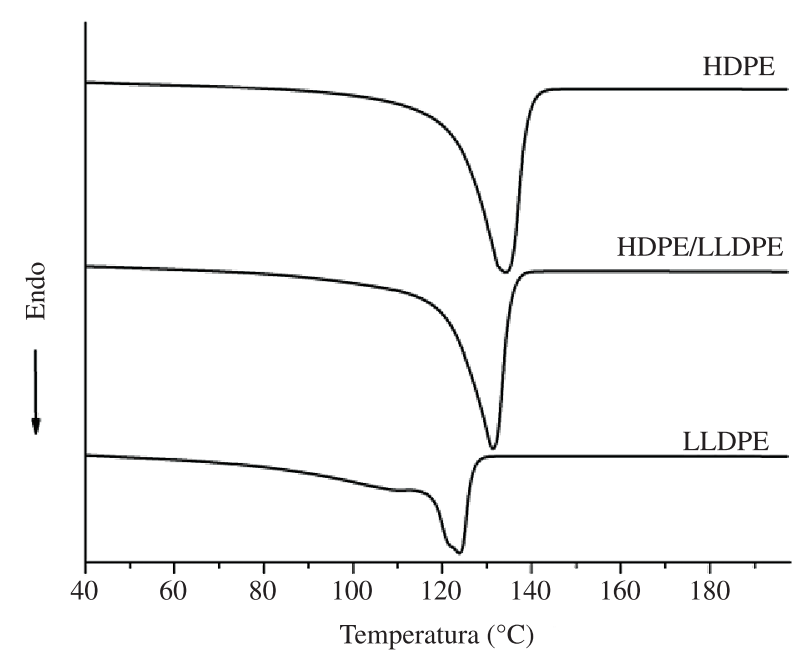

(b)

Figura 2. Termogramas de DSC do HDPE, LLDPE e blenda HDPE/LLDPE (75/25\%): a) resfriamento e b) segundo ciclo de aquecimento.

ambos os polímeros participam na formação do mesmo cristalito. A possibilidade de formação de blendas de polietileno que apresentam co-cristalização depende principalmente do número e do tamanho das ramificações ${ }^{[5,26]}$. O LLDPE utilizado possui 13 ramificações por 1000 átomos de carbono e estas são curtas (provenientes do buteno-1). Segundo Choi ${ }^{[2]}$, que utilizou o parâmetro de interação de Flory-Huggins para estudar o efeito do teor de ramificações do LLDPE na miscibilidade de blendas HDPE/LLDPE, a separação de fases pode ocorrer quando a quantidade de ramificações for igual ou superior a 40 ramificações por 1000 átomos de carbono; até esse valor, observou-se separação parcial de micro fases e acima de 60 ramificações foi observada separação total de fases. Portanto, espera-se também que por esse critério a blenda seja miscível.

\section{Caracterização estrutural}

A caracterização estrutural dos nanocompósitos obtidos através das diferentes sequências de mistura foi realizada através de ensaios de difração de raio X de alto ângulo (WAXD), microscopia eletrônica de transmissão (MET) e caracterização reológica a baixas taxas de cisalhamento. Os resultados das análises por WAXD da nanoargila e dos nanocompósitos obtidos por diferentes sequências de mistura são apresentados na Figura 3.

Os valores dos espaçamentos basais das amostras, calculados através da Lei de Bragg, encontram-se na Tabela 2. A ocorrência do processo de intercalação das cadeias poliméricas entre as camadas do silicato pode ser observada através do deslocamento do pico de difração $\left(\begin{array}{lll}0 & 0 & 1\end{array}\right)$ característico da nanoargila (em $2 \theta=3,8^{\circ}$, correspondendo a uma distância basal de 2,32 nm) para ângulos menores, já que o processo de intercalação leva a um aumento da distância basal da argila. Além do deslocamento do pico de difração, notou-se também um alargamento desses picos, sendo mais um indicativo do processo de intercalação parcial. Além do plano $\left(\begin{array}{lll}0 & 0 & 1\end{array}\right)$, pode-se observar um segundo ombro $\left(2 \theta=7,2^{\circ}\right)$ para a nanoargila que pode ser atribuído a distância $\mathrm{d}_{002}$ que é um plano paralelo ao $\mathrm{d}_{001}$. De maneira análoga, há um deslocamento proporcional desse ombro com a adição do sistema compatibilizante nos nanocompósitos. Dentre as sequências de mistura estudadas, M2 e M5 apresentaram maior distância basal. Nestas sequências, a nanoargila primeiramente foi misturada com elementos de menor viscosidade e cristalinidade (LLDPE e/ou LLDPE-g-MA), o que pode ter facilitado o processo de intercalação, uma vez que para

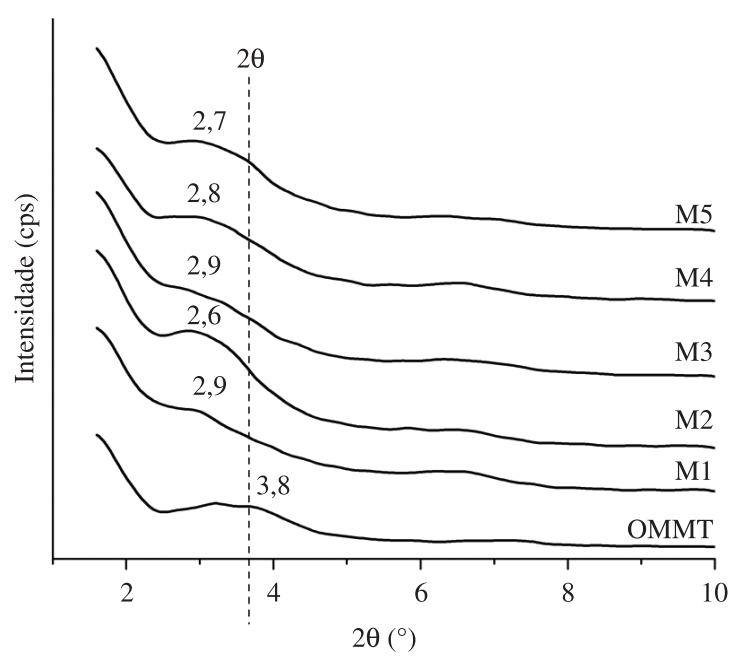

Figura 3. Difratogramas de raio $X$ da nanoargila e dos nanocompósitos obtidos por diferentes sequências de mistura.

Tabela 2. Valores do espaçamento basal $\left(d_{001}\right)$, grau de cristalinidade e tamanho aparente de cristalito das amostras estudadas.

\begin{tabular}{cccc}
\hline Composições & $\mathbf{d}_{\mathbf{0 0 1}}(\mathbf{n m})$ & $\begin{array}{c}\text { Cristalinidade } \\
(\mathbf{\%})\end{array}$ & $\begin{array}{c}\text { Tamanho de } \\
\text { cristalito }(\mathbf{n m})\end{array}$ \\
\hline HDPE & --- & 72,9 & 52 \\
LLDPE & --- & 54,1 & 38 \\
HDPE/LLDPE & --- & 63,1 & 48 \\
M1 & 3,07 & 61,8 & 48 \\
M2 & 3,42 & 61,6 & 42 \\
M3 & 3,07 & 68,3 & 50 \\
M4 & 3,17 & 64,5 & 55 \\
M5 & 3,30 & 61,2 & 47 \\
\hline
\end{tabular}

polímeros semicristalinos o processo de intercalação deve ocorrer preferencialmente na fase amorfa.

A Figura 4 apresenta os difratogramas de raio $X$ na região de altos ângulos para os materiais puros, a blenda e os nanocompósitos. O grau de cristalinidade e o tamanho aparente de cristalito foram calculados a partir da deconvolução matemática dos difratogramas de raio X e são apresentados na Tabela 2. 
Analisando os difratogramas, observa-se a presença de dois planos cristalográficos bem definidos $\left(\begin{array}{lll}1 & 1 & 0\end{array}\right)$ e $\left(\begin{array}{lll}2 & 0 & 0\end{array}\right)$ para todas as amostras estudadas. A posição dos picos relativos aos polímeros puros indica que o polietileno cristaliza nestas condições em estrutura ortorrômbica, e essa estrutura é mantida após a mistura do HDPE e LLDPE no estado fundido, tais resultados reforçam a teoria de ocorrência de co-cristalização na blenda estudada. No que concerne ao grau de cristalinidade da blenda polimérica, observa-se que este diminui proporcionalmente a quantidade de LLDPE adicionada ao sistema. No caso dos nanocompósitos observou-se que para as sequências M1, M2 e M5 ocorreu diminuição no grau de cristalinidade e do tamanho do cristalito quando comparado a blenda HDPE/LLDPE. O tamanho de cristalito está relacionado com o teor e tipo de ramificação do polímero. Quanto maior o número de ramificações, maior deve ser a separação das lamelas, criando regiões com maior quantidade de material amorfo, compostas pelas ramificações e moléculas de interligação lamelar, dificultando a cristalização, com isso reduzindo o tamanho dos cristalitos, como pode ser observado para os materiais puros, onde o HDPE possui cristalitos com tamanho aparente de $52 \mathrm{~nm}$ enquanto que o LLDPE, com maior número de ramificações curtas, possui tamanho de cristalito na ordem de $38 \mathrm{~nm}$. No caso da blenda polimérica, considerando ainda a heterogeneidade intermolecular entre HDPE

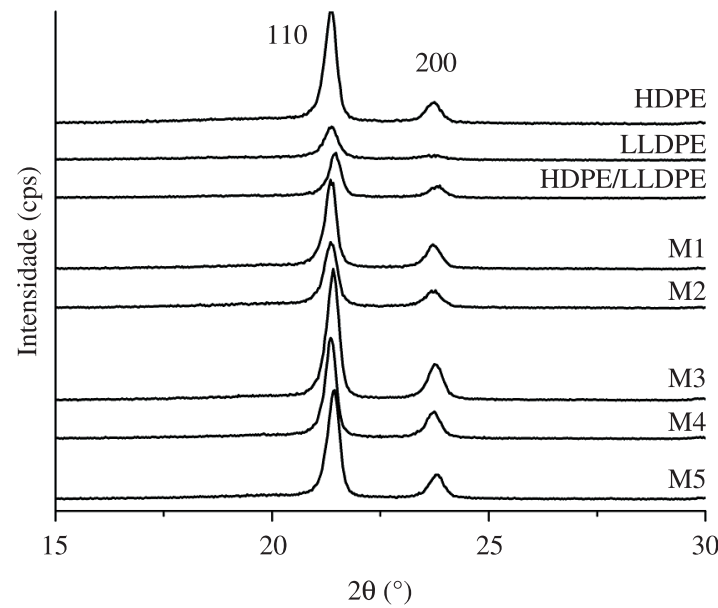

Figura 4. Difratogramas de raio $X$ na região de altos ângulos das amostras puras, da blenda HDPE/LLDPE e dos nanocompósitos obtidos a partir de diferentes sequências de mistura.

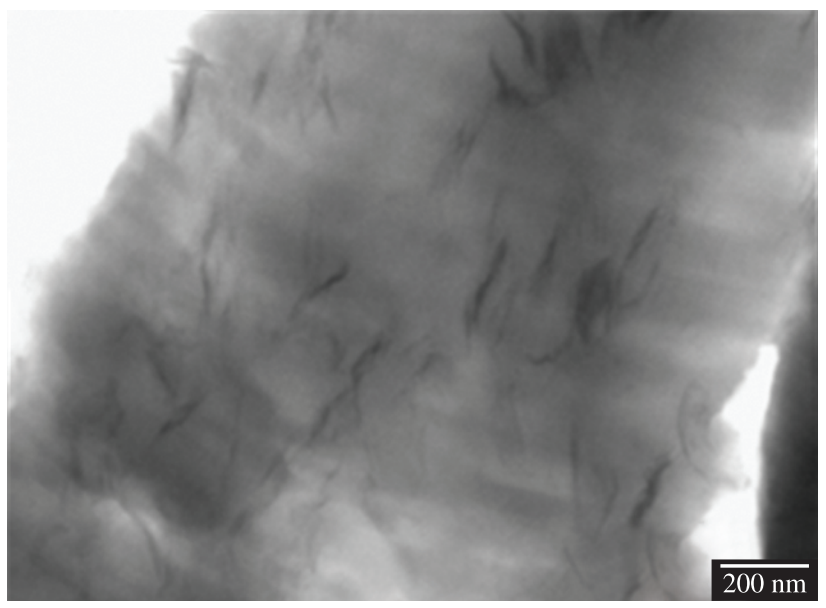

(a) e LLDPE (principalmente pela presença de maior número de ramificações curtas), no domínio rico de uma macromolécula, o arranjo regular dos segmentos de cadeias de outras espécies poderiam ser excluídos durante a cristalização e o estado original termodinâmico cristalino dessa macromolécula ser alterado, como resultado o tamanho do cristal torna-se menor.

O processo de intercalação e/ou esfoliação ocorre a medida que haja difusão das macromoléculas do agente compatibilizante ou da matriz polimérica nos espaços interlamelares. Esse processo dependerá de fatores eletrostáticos (polaridade relativa argila/polímero) e de fatores estéricos (entrópicos relacionados com a conformação dos segmentos de cadeia do polímero e consequentemente do volume molecular relativo ao espaço interlamelar). Por outro lado, a viscosidade do meio também possui um papel importante na mobilidade das moléculas para que a difusão ocorra ainda durante o tempo de mistura e desta forma, hipoteticamente, espera-se que os polímeros de menor viscosidade, LLDPE-g-MA e LLDPE, tenham favorecido sua difusão nos espaços interlamelares. Além disso, como é sabido que o processo de intercalação ocorre preferencialmente na fase amorfa, então a redução na cristalinidade também deverá favorecer o processo de intercalação e/ou esfoliação.

Uma vez que a blenda polimérica é miscível e ocorre co-cristalização, a sequência de mistura dos componentes do nanocompósito não tem como principal objetivo localizar preferencialmente a nanoargila em uma fase do sistema, mas determinar qual o melhor protocolo de mistura para alcançar o estado intercalado e a melhor dispersão das lamelas de silicato na matriz polimérica.

A morfologia e o estado de dispersão da argila nos nanocompósitos foram avaliados por microscopia eletrônica de transmissão. As Figuras 5 e 6 apresentam as micrografias obtidas das sequências M2 e M5, respectivamente. Essas sequências foram escolhidas por possuirem maior distância basal, menor grau de cristalinidade e tamanho de cristalito. Na micrografia, a fase mais escura representa a fase inorgânica de OMMT. Nota-se que a nanoargila encontra-se bem dispersa por toda a matriz, sendo possível observar a presença da estrutura intercalada, corroborando com os resultados de WAXD.

Comparando as micrografias das sequências M2 e M5 é possível observar que a sequência M2 possui estrutura mais homogênea com presença de grande número de lamelas intercaladas e presença de algumas camadas de silicato esfoliadas.

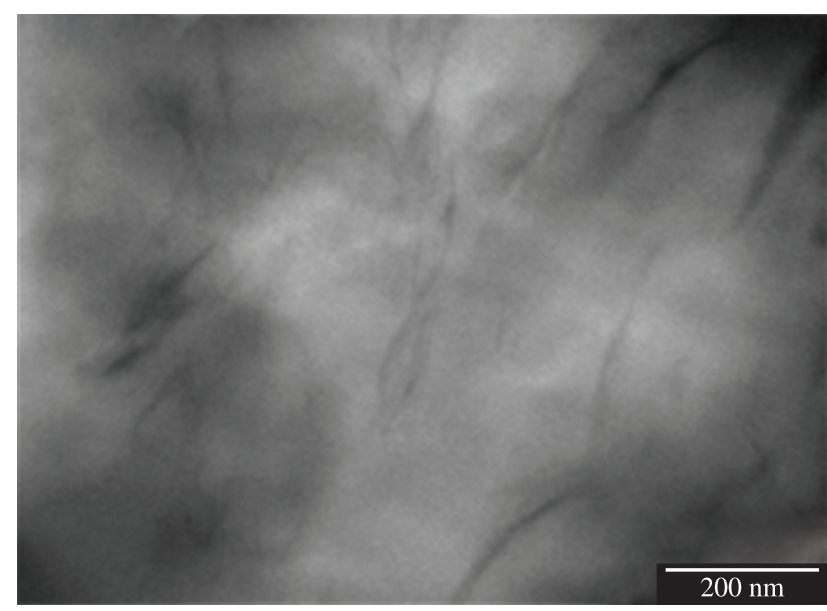

(b)

Figura 5. Micrografias obtidas por MET da sequência de mistura M2 - (LLDPE + LLDPE-g-MA+OMMT) + HDPE + HDPE-g-MA: a) 53.000× e b) 88.000×. 
Nos sistemas estudados, a utilização de mistura de dois agentes compatibilizantes enxertados com anidrido maleico, miscíveis com os constituintes da matriz, auxiliou na obtenção de uma distribuição de grupos polares pela matriz polimérica facilitando a dispersão das nanoargilas por toda a matriz. $\mathrm{O}$ estudo das interações entre os constituintes dos nanocompósitos foi realizado através do comportamento reológico em regime permanente a baixas taxas de cisalhamento. A Figura 7 apresenta as curvas de viscosidade em função da taxa de cisalhamento das amostras estudadas.

Analisando as curvas de viscosidade em função da taxa de cisalhamento, pode-se observar que o LLDPE possui um comportamento predominante Newtoniano com viscosidade bem inferior aos demais sistemas estudados enquanto que o HDPE possui um pequeno platô Newtoniano. A adição da nanoargila aumentou a viscosidade a baixas taxas de cisalhamento quando comparados à blenda HDPE/LLDPE, alterando o comportamento Newtoniano para um comportamento pseudoplástico mais pronunciado. Em nanocompósitos com estrutura altamente interpenetrantes ou conectadas o comportamento de Binghan pode ser observado, necessitando-se aplicar uma alta tensão para que se verifique fluxo. Essa mudança pode ser atribuída às interações friccionais entre as camadas de silicato e não devido a imobilização das cadeias poliméricas confinadas entre as camadas de silicato, além da presença de interações entre os grupos polares do compatibilizante e o modificador orgânico da OMMT ${ }^{[27]}$.

Os polímeros de maneira geral seguem a Regra de Cox-Merz, porém essa regra não é válida para nanocompósitos, uma vez que quando submetidos a fluxos elongacionais as lamelas tendem a se alinhar de maneira perpendicular ao fluxo ${ }^{[10,28]}$. Estudos com nanocompósitos de poli(butileno tereftalato) com argila ${ }^{[29]}$ concluíram que é possível correlacionar o valor do índice da Lei das Potências, $n$, com o grau de dispersão da nanoargila na matriz

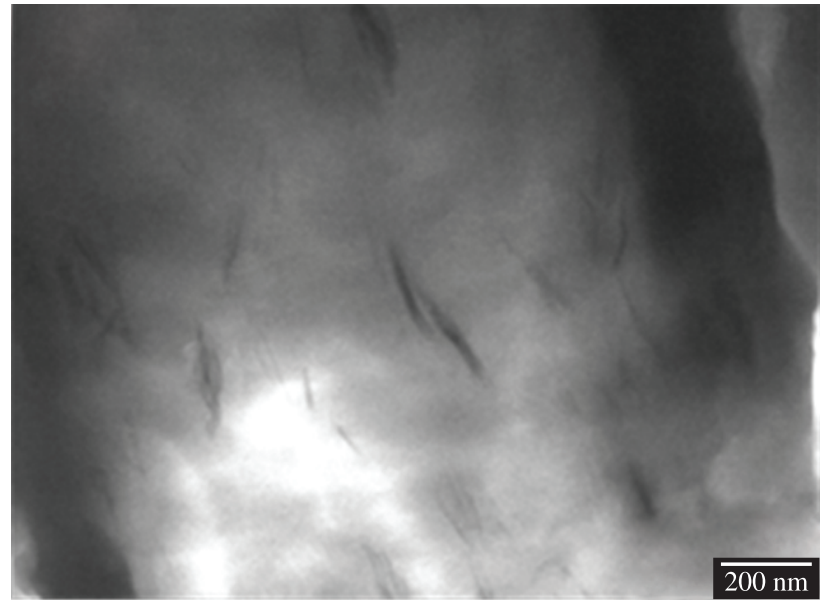

(a)

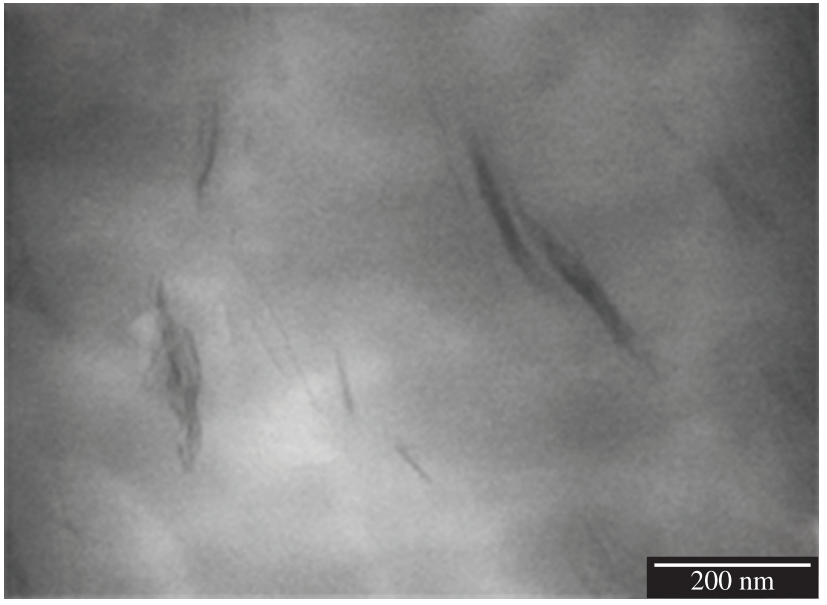

(b)

Figura 6. Micrografias obtidas por MET da sequência de mistura M5 - (HDPE-g-MA+LLDPE-g-MA+OMMT) + HDPE + LLDPE: a) 53.000× e b) 88.000×.

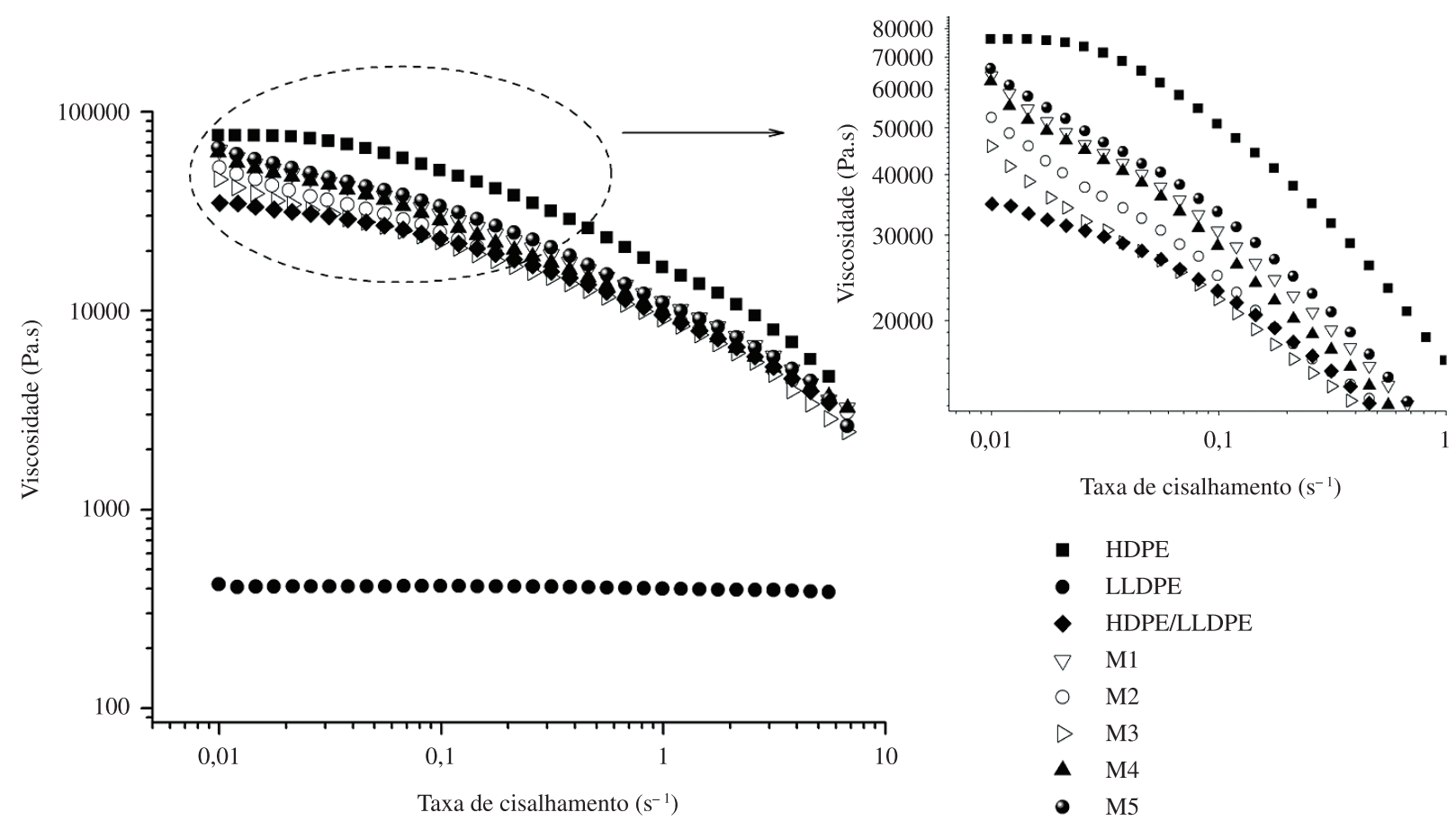

Figura 7. Viscosidade em função da taxa de cisalhamento dos materiais puros, da blenda HDPE/LLDPE (75/25\%) e dos nanocompósitos obtidos a partir de diferentes sequências de mistura. Ensaios realizados em $180^{\circ} \mathrm{C}$. 
polimérica. Pode-se assumir que, em baixas taxas de cisalhamento, a viscosidade desses materiais pode ser dada pela Equação 3.

$$
\eta=A \cdot \gamma^{m}
$$

onde $\mathrm{m}$ é a inclinação da curva $\log \eta$ em função de $\log \gamma$ e A é uma constante. Quando $\mathrm{m} \rightarrow 0$, a viscosidade tende a um valor constante (comportamento Newtoniano); já quando $\mathrm{m} \rightarrow \infty$ ou -1 , o comportamento é similar ao de um sólido elástico. Para a blenda HDPE/LLDPE o valor de $\mathrm{m}$ é igual a -0,21; a adição de argila diminui o valor de $\mathrm{m}$, sendo obtido menor valor para as sequências M2 $(\mathrm{m}=-0,49)$ e M5 $(\mathrm{m}=-0,50)$, indicando que nestas sequências de mistura há interações mais intensas entre a matriz e a nanoargila quando comparadas as demais sequências estudadas (M1: $\mathrm{m}=-0,47 ; \mathrm{M} 3: \mathrm{m}=-0,42$ e $\mathrm{M} 4: \mathrm{m}=-0,43$ ).

Estes resultados corroboram com os resultados obtidos por difração de raio X, e evidencia a importância do estudo da sequência de mistura na busca de maiores interações entre a matriz orgânica e a carga inorgânica. Além da sequência de mistura outros fatores são muito importantes para a produção de nanocompósitos intercalados e/ou esfoliados de polietileno/argila, como a influencia do cisalhamento na obtenção dos nanocompósitos e a compatibilidade da matriz polimérica e da carga inorgânica ${ }^{[30]}$, as forças de cisalhamento facilitam a quebra dos aglomerados grandes, enquanto que a extensão da esfoliação das nanocargas é determinada mais pela compatibilidade entre a matriz polimérica e as camadas da argila, do que pelas forças de cisalhamento. Como neste estudo a matriz polimérica foi constituída pela mistura de dois polímeros, buscou-se alcançar o estado intercalado com a utilização de dois agentes compatibilizantes, compatíveis e miscíveis com os respectivos componentes da blenda polimérica. A sequência de mistura influenciou no grau de cristalinidade e consequentemente no comportamento reológico dos nanocompósitos, sendo obtido maior grau de intercalação/esfoliação nos sistemas em que primeiramente a argila foi misturada com constituintes de menor viscosidade.

\section{Conclusões}

Neste trabalho, nanocompósitos de blendas de HDPE/LLDPE compatibilizadas com uma mistura de HDPE-g-MA/LLDPE-g-MA foram estudados. Estudo de DMA e DSC evidenciaram a formação de um sistema miscível e ocorrência de co-cristalização entre o HDPE e o LLDPE. Dessa forma a sequência de mistura foi utilizada para alcançar o estado intercalado e a melhor dispersão das lamelas de silicato na matriz polimérica. $\mathrm{O}$ uso de um sistema compatibilizante composto por mistura de dois agentes enxertados com anidrido maleico, miscíveis com os constituintes da matriz, auxiliou na distribuição da polaridade facilitando a dispersão das nanoargilas por toda a matriz. Pode-se concluir que sequências em que a nanoargila foi primeiramente misturada com constituintes de menor viscosidade e cristalinidade (LLDPE e/ou LLDPE-g-MA) foram as mais eficazes para a formação da estrutura intercalada nos nanocompósitos, uma vez que para polímeros semicristalinos o processo de intercalação deve ocorrer preferencialmente na fase amorfa.

\section{Agradecimentos}

Os autores agradecem ao $\mathrm{CNPq}$ e a FAPESP pelo apoio financeiro.

\section{Referências Bibliográficas}

1. Liu, C.; Wang, J. \& He, J. - Polymer, 43, p.3811 (2002). http://dx.doi. org/10.1016/S0032-3861(02)00201-X

2. Choi, P. - Polymer, 41, 8741 (2000). http://dx.doi.org/10.1016/S00323861(00)00279-2
3. Munaro, M. \& Akcelrud, L. - Polym. Degrad. Stab., 93, p.43 (2008). http://dx.doi.org/10.1016/j.polymdegradstab.2007.10.017

4. Lee, S. Y. \& Jho, J. Y. - J. Ind. Eng. Chem., 4, p.170 (1998).

5. Liang, S.; Yang, H.; Wang, K.; Zhang, Q.; Du, R. \& Fu, Q. - Acta Mat., 56, p.50 (2008). http://dx.doi.org/10.1016/j.actamat.2007.09.008

6. Morales, A. R.; Cruz, C.; Peres, L. \& Ito, E. N. - Polímeros, 20, p.39 (2010). http://dx.doi.org/10.1590/S0104-14282010005000004

7. Alexandre, M.; Dubois, P.; Sun, T.; Garces, J. M. \& Jérome, R. - Polymer, 43, p.2123 (2002). http://dx.doi.org/10.1016/S00323861(02)00036-8

8. Ray, S. S. \& Okamoto, M. - Progr. Polym. Sci., 28, p.1539 (2003).

9. Alexandre, M. \& Dubois, P. - Mat. Sci. Eng., 28, p.1 (2000). http:// dx.doi.org/10.1016/S0927-796X(00)00012-7

10. Fornes, T. D.; Yoon, P. J.; Keskkula, H. \& Paul, D. R. - Polymer, 42, p.9929 (2001). http://dx.doi.org/10.1016/S0032-3861(01)00552-3

11. Heinemann, J.; Reichert, P.; Thomann, R. \& Mulhaupt, R. - Macromol. Rapid Commun, 20, p.423 (1999). http://dx.doi.org/10.1002/ (SICI)1521-3927(19990801)20:8<423::AID-MARC423>3.0.CO;2-N

12. Jin, Y. H.; Park, H. J.; Im, S. S.; Kwak, S. Y. \& Kwak S. - Macromol. Rapid Commun, 23, p.135 (2002). http://dx.doi.org/10.1002/1521-392 7(20020101)23:2<135::AID-MARC135>3.0.CO;2-T

13. Hotta, S. \& Paul, D. R. - Polymer, 45, p.7639 (2004). http://dx.doi. org/10.1016/j.polymer.2004.08.059

14. Gopakumar, T. G.; Lee, J. A.; Kontopoulou, M. \& Parent, J, S. - Polymer, 43, p.5483 (2002). http://dx.doi.org/10.1016/S00323861(02)00403-2

15. Wang, K. H.; Choi, M. H.; Koo, C. M.; Choi, Y. S. \& Chung, I. J. - Polymer, 42, p.9819 (2001). http://dx.doi.org/10.1016/S00323861(01)00509-2

16. Spencer, M. W.; Cui, L.; Yoo, Y. \& Paul, D. R. - Polymer, 51, p.1056 (2010). http://dx.doi.org/10.1016/j.polymer.2009.12.047

17. Dasari, A.; Yu, Z. Z. \& Mai, Y. W. - Polymer, 46, p.5986 (2005). http:// dx.doi.org/10.1016/j.polymer.2005.05.145

18. Martins, C. G.; Larocca, N. M.; Paul, D. R. \& Pessan, L. A. - Polymer, 50, p.1743 (2009). http://dx.doi.org/10.1016/j.polymer.2009.01.059

19. Choi, J. H.; Kim, H.; Han, D. H. \& Lim, J. C. - J. Appl. Polym Sci, 101, p.1 (2006). http://dx.doi.org/10.1002/app.22081

20. Fan, Y.; Xue, Y.; Nie, W.; Ji, X. \& Bo, S. - Polym. J., 41, p.622 (2009). http://dx.doi.org/10.1295/polymj.PJ2009030

21. Spevacek, J. - Polymer, 19, p.1149 (1978).

22. Passador, F. R.; Rodolfo Junior, A. \& Pessan, L. A. - Polímeros, 16, p.174 (2006). http://dx.doi.org/10.1590/S0104-14282006000300005

23. Quental, A. C.; Carvalho, F. P.; Tada, E. S. \& Felisberti, M. I. - Quím. nova, 33, p.438 (2010). http://dx.doi.org/10.1590/S010040422010000200035

24. Fox, T. G. - Bull. Am. Phys. Soc., 1, p.123 (1956).

25. Hussein, I. A. - Macromolecules, 36, p.2024 (2003). http://dx.doi. org $/ 10.1021 / \mathrm{ma} 0257245$

26. Ranade, A.; Nayak, K.; Fairbrother, D. \& D'souza, N. A. - Polymer, 46, p.7323 (2005). http://dx.doi.org/10.1016/j.polymer.2005.04.085

27. Okamoto, M.; Nam, P. H.; Manti, P.; Kotaka, T.; Hasegawa, N. \& Usuki, A. - Nano lett., 1, p.285 (2001).

28. Wagener, R. \& Rusenger, T. J. G. - Polymer, 44, p.7513 (2003). http:// dx.doi.org/10.1016/j.polymer.2003.01.001

29. Homminga, D.; Goderis, B.; Hoffman, S.; Reynaers, H. \& Groeninckx, G. - Polymer, 46, p.9941 (2005). http://dx.doi.org/10.1016/j. polymer.2005.07.059

Enviado: 20/06/11

Reenviado: $27 / 09 / 11$

Aceito: $07 / 11 / 11$ 